\title{
Perfect and bipartite IMTL-algebras and disconnected rotations of prelinear semihoops
}

\author{
Carles Noguera \\ Institut d'Investigació en Intel-ligència Artificial - CSIC \\ Catalonia, Spain \\ Francesc Esteva \\ Institut d'Investigació en Intel-ligència Artificial - CSIC \\ Catalonia, Spain \\ Joan Gispert \\ Universitat de Barcelona \\ Catalonia, Spain
}

\begin{abstract}
IMTL logic was introduced in [12] as a generalization of the infinitely-valued logic of Łukasiewicz, and in [11] it was proved to be the logic of left-continuous t-norms with an involutive negation and their residua. The structure of such t-norms is still not known. Nevertheless, Jenei introduced in [20] a new way to obtain rotation-invariant semigroups and, in particular, IMTL-algebras and left-continuous t-norm with an involutive negation, by means of the disconnected rotation method. In order to give an algebraic interpretation to this construction, we generalize the concepts of perfect, bipartite and local algebra used in the classification of MV-algebras to the wider variety of IMTL-algebras and we prove that perfect algebras are exactly those algebras obtained from a prelinear semihoop by Jenei's disconnected rotation. We also prove that the variety generated by all perfect IMTL-algebras is the variety of the IMTL-algebras that are bipartite by every maximal filter and we give equational axiomatizations for it.

Keywords: Algebraizable logics, bipartite algebras, cancellative hoops, disconnected rotation, filters, IMTL-algebras, local algebras, many-valued logic, MV-algebras, perfect algebras, prelinear semihoops, Wajsberg hoops
\end{abstract}

\section{Introduction}

MV-algebras were introduced by C.C. Chang in $[5,6]$ as an algebraic tool to study the infinitely-valued logic of Eukasiewicz. They form a variety that gives an algebraic semantics for this multivalued logic. Furthermore, since Eukasiewicz logic is a finitary algebraizable logic in the sense of Blok and Pigozzi (see [3]), there is a correspondence between its axiomatic extensions and the subvarieties of MV-algebras. In fact, this lattice of subvarieties was intensively studied and finally fully described, obtaining thus a classification of all axiomatic extensions of Łukasiewicz logic (see [22]).

Some years after, some logics weaker than Łukasiewicz logic were introduced, namely the logic BL defined by Hájek in [18], and the logics MTL and IMTL defined by Esteva and Godo 
in [12]. In particular, IMTL proves, as Łukasiewicz logic, the law of involution of the negation, $\neg \neg \varphi \rightarrow \varphi$, and it is complete with respect to the semantics given by involutive left-continuous t-norms and their residua (see [11]). The structure of such t-norms is still not known. There are only partial results by Jenei giving some methods to construct left-continuous involutive t-norms. They are summarized in [21].

IMTL is also an algebraizable logic whose equivalent algebraic semantics, the variety of IMTL-algebras, contains the variety of MV-algebras. Therefore, the study of all axiomatic extensions of IMTL, or equivalenty the study of all subvarieties of IMTL-algebras, will be a generalization of the work done for MV-algebras. Some of these subvarieties have been studied in $[7,16,17]$.

In this paper we do a new step towards this aim, extending the work done in $[1,2,9]$ for the perfect, bipartite and local MV-algebras to IMTL-algebras. As it was done for BL-algebras in [10], we translate these notions into the language of IMTL using the multiplicative conjunction instead of the multiplicative disjunction and using the notion of filter instead of the notion of ideal, since the concept of filter is useful in the whole variety of MTL-algebras. Indeed, for MTL-algebras there is a correspondence between filters and congruences. This will help in extending the work done here for IMTL-algebras to all MTL-algebras in future research. We also use some of the methods introduced by S. Jenei in [20] for obtaining IMTL-algebras and put them in relation with the concept of perfect algebras.

\section{Preliminaries}

In this section we will introduce some definitions and facts needed for the discussion of the paper. For any unexplained notion on Universal Algebra see [4].

IMTL logic is presented in [12] by means of a Hilbert-style calculus in the language $\mathcal{L}=\{*, \rightarrow, \wedge, 0\}$ of type $(2,2,2,0)$. The only inference rule is Modus Ponens and the axiom schemata are the following (taking $\rightarrow$ as the least binding connective):

$$
\begin{array}{ll}
\text { (A1) } & (\varphi \rightarrow \psi) \rightarrow((\psi \rightarrow \chi) \rightarrow(\varphi \rightarrow \chi)) \\
\text { (A2) } & \varphi * \psi \rightarrow \varphi \\
\text { (A3) } & \varphi * \psi \rightarrow \psi * \varphi \\
\text { (A4) } & \varphi \wedge \psi \rightarrow \varphi \\
\text { (A5) } & \varphi \wedge \psi \rightarrow \psi \wedge \varphi \\
\text { (A6) } & \varphi *(\varphi \rightarrow \psi) \rightarrow \varphi \wedge \psi \\
\text { (A7a) } & (\varphi \rightarrow(\psi \rightarrow \chi)) \rightarrow(\varphi * \psi \rightarrow \chi) \\
\text { (A7b) } & (\varphi * \psi \rightarrow \chi) \rightarrow(\varphi \rightarrow(\psi \rightarrow \chi)) \\
\text { (A8) } & ((\varphi \rightarrow \psi) \rightarrow \chi) \rightarrow(((\psi \rightarrow \varphi) \rightarrow \chi) \rightarrow \chi) \\
\text { (A9) } & 0 \rightarrow \varphi \\
\text { (A10) } & \neg \neg \varphi \rightarrow \varphi
\end{array}
$$

The usual defined connectives are introduced as follows:

$$
\begin{aligned}
& \varphi \vee \psi:=((\varphi \rightarrow \psi) \rightarrow \psi) \wedge((\psi \rightarrow \varphi) \rightarrow \varphi) ; \\
& \varphi \leftrightarrow \psi:=(\varphi \rightarrow \psi) *(\psi \rightarrow \varphi) ;
\end{aligned}
$$




$$
\begin{aligned}
& \neg \varphi:=\varphi \rightarrow 0 ; \\
& \varphi \oplus \psi:=\neg(\neg \varphi * \neg \psi) ; \\
& 1:=\neg 0 .
\end{aligned}
$$

We denote the set of $\mathcal{L}$-formulas by $F m_{\mathcal{L}}$. Given $\Gamma \cup\{\varphi\} \subseteq F m_{\mathcal{L}}$ we write $\Gamma \vdash_{I M T L} \varphi$ if $\varphi$ is provable from $\Gamma$ in the system IMTL.

In order to give an algebraic semantics for IMTL, the following algebras are also introduced in $[12]$ :

Definition 2.1 ([12]). Let $\mathcal{A}=\langle A, *, \rightarrow, \wedge, \vee, 0,1\rangle$ be an algebra of type $(2,2,2,2,0,0)$. We define a unary operation by $\neg a:=a \rightarrow 0$. $\mathcal{A}$ is an IMTL-algebra if it is a bounded residuated lattice satisfying the prelinearity equation:

$$
(x \rightarrow y) \vee(y \rightarrow x) \approx 1,
$$

and the involution equation:

$$
\neg \neg x \approx x .
$$

The algebra has negation fixpoint if there is $a \in A$ such that $\neg a=a$. In [19] it is proved that there is at most one fixpoint.

If the lattice order is total we will say that $\mathcal{A}$ is an IMTL-chain.

$\mathbb{I M T L}$ will denote the variety of all IMTL-algebras.

Definition 2.2. Let $E q_{\mathcal{L}}$ be the set of $\mathcal{L}$-equations, i.e. the set of expressions of the form $\varphi \approx \psi$ where $\varphi, \psi \in F m_{\mathcal{L}}$. Let $\Sigma \cup\{\varphi \approx \psi\} \subseteq E q_{\mathcal{L}}$. We define:

$\Sigma \vDash_{\mathbb{I M T L}} \varphi \approx \psi$ if for every $\mathcal{A} \in \mathbb{I M T L}$ and every evaluation $v$ in $\mathcal{A}$, it holds:

$$
\text { If for every } \alpha \approx \beta \in \Sigma, v(\alpha)=v(\beta) \text {, then } v(\varphi)=v(\psi) \text {. }
$$

Now, having this notion of equational consequence, we can state the strong link existing between the logic IMTL and the variety IMTL:

Theorem 2.3. The relation of derivability in IMTL and the equational consequence determined by the variety $\mathbb{I M T L}$ are mutually translatable. Indeed, given $\Gamma \cup\{\gamma\} \subseteq F m_{\mathcal{L}}$ and $\Sigma \cup\{\varphi \approx \psi\} \subseteq E q_{\mathcal{L}}$, we have:

1. $\Gamma \vdash_{I M T L} \gamma$ iff $\{\psi \approx 1: \psi \in \Gamma\} \vDash_{\mathbb{M M T L}} \gamma \approx 1$.

2. $\Sigma \vDash_{\mathbb{I M T L}} \varphi \approx \psi$ iff $\{\alpha \leftrightarrow \beta: \alpha \approx \beta \in \Sigma\} \vdash_{I M T L} \varphi \leftrightarrow \psi$.

Moreover, each one of these translations is the inverse of the other one in the following sense:

3. $\varphi \approx \psi \vDash_{\mathbb{M M T L}} \varphi \leftrightarrow \psi \approx 1$ and $\varphi \leftrightarrow \psi \approx 1 \vDash_{\mathbb{M M T L}} \varphi \approx \psi$.

4. $\gamma \vdash_{I M T L} \gamma \leftrightarrow 1$ and $\gamma \leftrightarrow 1 \vdash_{I M T L} \gamma$. 
In the terminology of [3] this means that IMTL is an algebraizable logic whose equivalent algebraic semantics is IMTL. As a consequence, there is an order-reversing isomorphism between the axiomatic extensions of IMTL and the subvarieties of $\mathbb{I M T L}$, given by the translations of the above theorem.

Some IMTL-algebras are well-known. For instance, Boolean algebras are those IMTLalgebras where the equation of the excluded middle, $x \vee \neg x \approx 1$, is valid, and MV-algebras are those IMTL-algebras satisfying the equation of divisibility $x \wedge y \approx x *(x \rightarrow y)$. Another interesting class of IMTL-algebras are the so-called NM-algebras. They were defined in [12] as those IMTL-algebras satisfying the following equation:

$$
(x * y \rightarrow 0) \vee(x \wedge y \rightarrow x * y) \approx 1
$$

There is, up to isomorphism, only one NM-algebra defined over the real unit interval $[0,1]$, given by the nilpotent minimum t-norm (introduced by Fodor in [14]):

$$
a *_{N M} b= \begin{cases}\min \{a, b\} & \text { if } a>1-b, \\ 0 & \text { otherwise. }\end{cases}
$$

and its residuated implication:

$$
a \rightarrow_{N M} b= \begin{cases}1 & \text { if } a \leq b, \\ \max \{1-a, b\} & \text { otherwise }\end{cases}
$$

We will denote this algebra as $[0,1]_{N M}$. It generates the whole variety of NM-algebras $([12])$.

For a general study of IMTL-algebras we need some definitions.

Definition 2.4. If $\mathcal{A}$ is an IMTL-algebra, the sets of positive and negative elements are respectively defined as:

$A_{+}:=\{a \in A: a>\neg a\}$

$A_{-}:=\{a \in A: a \leq \neg a\}$

The following characterization of these sets will be useful.

Proposition 2.5. Let $\mathcal{A}$ be an IMTL-algebra. Then:

$A_{+}=\{a \vee \neg a: a \in A, a \neq \neg a\}$

$A_{-}=\{a \wedge \neg a: a \in A\}$

Proof. If $a \in A_{+}$then $a=a \vee \neg a$. Conversely, if $a$ is not a fixpoint, we have $a \vee \neg a>$ $\neg(a \vee \neg a)=a \wedge \neg a$, since $a \vee \neg a \geq a, \neg a \geq a \wedge \neg a$. The other equality is provable with a similar reasoning.

Recall that a filter in an IMTL-algebra is any set $F$ such that:

- $1 \in F$.

- If $a \in F$ and $a \leq b$, then $b \in F$. 
- If $a, b \in F$, then $a * b \in F$.

$F$ is proper if $0 \notin F . F$ is a prime filter if it is proper and for every $a, b \in A$ if $a \vee b \in F$, then $a \in F$ or $b \in F$.

Using Zorn's Lemma one can prove that for any proper filter $F$ there is a maximal proper filter $G$ such that $F \subseteq G$. Moreover, every maximal filter is prime. The radical of $\mathcal{A}$ is defined as $\operatorname{Rad}(\mathcal{A})=\bigcap\{M \subseteq A: M$ is a maximal filter $\}$. Notice that in a chain the set of filters is totally ordered, hence the radical is the maximum proper filter.

Recall this known property of maximal filters:

Proposition 2.6. Let $\mathcal{A}$ be an IMTL-algebra and $M \subseteq A$ a maximal filter. Then for every $a \in A, a \notin M$ if, and only if, there exists $n$ such that $\neg a^{n} \in M$.

We state also, for the reader's convenience, the known correspondence between filters and congruences in IMTL-algebras.

Proposition 2.7. Let $\mathcal{A}$ be an IMTL-algebra. For every filter $F \subseteq A$ we define $\Theta(F):=$ $\left\{\langle a, b\rangle \in A^{2}: a \leftrightarrow b \in F\right\}$, and for every congruence $\theta$ of $\mathcal{A}$ we define $F i(\theta):=\{a \in A$ : $\langle a, 1\rangle \in \theta\}$. Then, $\Theta$ is an order isomorphism from the set of filters onto the set of congruences and $\mathrm{Fi}$ is its inverse.

Recall also that all IMTL-algebras are representable as a subdirect product of IMTLchains, as proved in [12].

Now, in order to introduce some methods for constructing IMTL-algebras defined by Jenei (see [20]), we need first to define the notion of prelinear semihoop.

Definition 2.8 ([13]). An algebra $\mathcal{A}=\left\langle A, *^{\mathcal{A}}, \rightarrow^{\mathcal{A}}, \wedge^{\mathcal{A}}, 1^{\mathcal{A}}\right\rangle$ of type $(2,2,2,0)$ is a semihoop if it satisfies the following conditions:

- $\mathcal{A}=\left\langle A, \wedge^{\mathcal{A}}, 1^{\mathcal{A}}\right\rangle$ is an inf-semilattice with upper bound.

- $\left\langle A, *^{\mathcal{A}}, 1^{\mathcal{A}}\right\rangle$ is a commutative monoid isotonic w.r.t. the inf-semilattice order.

- For every $a, b \in A, a \leq^{\mathcal{A}} b$ if, and only if, $a \rightarrow^{\mathcal{A}} b=1^{\mathcal{A}}$.

- For every $a, b, c \in A a *^{\mathcal{A}} b \rightarrow^{\mathcal{A}} c=a \rightarrow^{\mathcal{A}}\left(b \rightarrow^{\mathcal{A}} c\right)$.

If in addition it satisfies the prelinearity equation, it is a prelinear semihoop. ${ }^{1}$

$A$ semihoop $\mathcal{A}$ is a hoop if $\mathcal{A}=x *(x \rightarrow y) \approx y *(y \rightarrow x)$.

$A$ hoop $\mathcal{A}$ is cancellative if $a *^{\mathcal{A}} b \leq c *^{\mathcal{A}} b$ implies $a \leq{ }^{\mathcal{A}} c$, for every $a, b, c \in A$.

$A$ Wajsberg hoop is a hoop satisfying $(x \rightarrow y) \rightarrow y \approx(y \rightarrow x) \rightarrow x$.

In [13] is proved that cancellative hoops coincide with unbounded Wajsberg hoops.

\footnotetext{
${ }^{1}$ In [13] that kind of semihoops are called basic semihoops but here we prefer to use the more descriptive adjective prelinear.
} 
Definition 2.9. Let $\mathcal{A}$ be a prelinear semihoop. We define an algebra, $\mathcal{A}^{*}$, called the disconnected rotation of $\mathcal{A}$. Let $A \times\{0\}$ be a disjoint copy of $A$. For every a $\in A$ we write $a^{\prime}$ instead of $\langle a, 0\rangle$. Consider $\left\langle A^{\prime}=\left\{a^{\prime}: a \in A\right\}, \leq\right\rangle$ with the inverse order and let $A^{*}:=A \cup A^{\prime}$. We extend these orderings to an order in $A^{*}$ by putting $a^{\prime}<b$ for every $a, b \in A$. Finally, we take the following operations in $\mathcal{A}^{*}$ :

$1^{\mathcal{A}^{*}}:=1^{\mathcal{A}}, 0^{\mathcal{A}^{*}}:=\left(1^{\mathcal{A}}\right)^{\prime}, \wedge^{\mathcal{A}^{*}}$ the minimum w.r.t. the ordering, $\vee^{\mathcal{A}^{*}}$ the maximum w.r.t. the ordering,

$$
\begin{aligned}
& \neg^{\mathcal{A}^{*}} a:=\left\{\begin{array}{lll}
a^{\prime} & \text { if } & a \in A \\
b & \text { if } & a=b^{\prime} \in A^{\prime}
\end{array}\right. \\
& a * \mathcal{A}^{*} b:=\left\{\begin{array}{lll}
a *^{\mathcal{A}} b & \text { if } & a, b \in A \\
\mathcal{A}^{*}\left(a \rightarrow \mathcal{A} \neg \mathcal{A}^{*} b\right) & \text { if } & a \in A, b \in A^{\prime} \\
\mathcal{A}^{*}\left(b \rightarrow \mathcal{A} \mathcal{A}^{*} a\right) & \text { if } & a \in A^{\prime}, b \in A \\
0^{\mathcal{A}^{*}} & \text { if } & a, b \in A^{\prime}
\end{array}\right. \\
& a \rightarrow \mathcal{A}^{*} b:=\left\{\begin{array}{lll}
a \rightarrow{ }^{\mathcal{A}} b & \text { if } & a, b \in A \\
\mathcal{A}^{*}\left(a * \mathcal{A} \neg \mathcal{A}^{*} b\right) & \text { if } & a \in A, b \in A^{\prime} \\
\mathcal{A}^{*} & \text { if } & a \in A^{\prime}, b \in A \\
\mathcal{A}^{*} b \rightarrow \mathcal{A} \neg \mathcal{A}^{*} a & \text { if } & a, b \in A^{\prime}
\end{array}\right.
\end{aligned}
$$

Proposition 2.10 ([20]). Disconnected rotations are IMTL-algebras without fixpoint.

\section{Main results}

Let $\mathcal{A}$ be an IMTL-algebra.

Definition 3.1. For $a \in A$ we define $a^{0}:=1, a^{1}:=a$ and for every $n>1 a^{n}:=a^{n-1} * a$. Using this notation we can define the order of $a \in A$ as:

$$
\operatorname{ord}(a)= \begin{cases}\min \left\{n: a^{n}=0\right\} & \text { if it exists } \\ \infty & \text { otherwise }\end{cases}
$$

Note that:

- $\operatorname{ord}(1)=\infty$.

- If $F$ is a proper filter and $a \in F$, then $\operatorname{ord}(a)=\infty$.

- If $a \in A_{-}$, then $\operatorname{ord}(a)<\infty$.

The definition of order gives rise to the notion of perfect algebra.

Definition 3.2. $\mathcal{A}$ is perfect if for every $a \in A, \operatorname{ord}(a)<\infty$ if, and only if, $\operatorname{ord}(\neg a)=\infty$.

Some easy examples of perfect IMTL-algebras are the Boolean algebra of two elements $\mathcal{B}_{2}$, the Chang MV-algebra defined in [5] and all NM-chains without negation fixpoint. In all these algebras one can check that all positive elements have infinite order.

We also want to extend the notion of bipartite MV-algebra to the class of all IMTLalgebras. To this end, we need a previous proposition. 
Proposition 3.3. Let $F \subseteq A$ be a filter of $\mathcal{A}$. Then the subuniverse of $A$ generated by $F$ is $F \cup \neg F$, where $\neg F=\{\neg a: a \in F\}$.

Proof. Let $X$ be the subuniverse of $A$ generated by $F$. We must see that $X=F \cup \neg F$. The inclusion from right to left is obvious, so we only prove the other one. Note that $F \subseteq F \cup \neg F$, thus it is enough to show that $F \cup \neg F$ is a subuniverse. This is easily done by proving that it is closed under all the operations.

Definition 3.4. $\mathcal{A}$ is bipartite if there is a maximal filter $F \subseteq A$ such that $A=F \cup \neg F$, i.e., such that $F$ generates $\mathcal{A}$. In this case we will say that $\mathcal{A}$ is bipartite by $F$.

The filter $F$ in the previous definition need not to be unique; it is possible to have an algebra bipartite by more than one maximal filter. In fact, we define a special class of bipartite IMTL-algebras, $\mathbb{B P}_{0}$, as those that are bipartite by all maximal filters.

Definition 3.5. $\mathcal{A} \in \mathbb{B P}_{0}$ if for every maximal filter $F \subseteq A, A=F \cup \neg F$.

All the examples given above of perfect algebras are also algebras of $\mathbb{B P}_{0}$. But not all the algebras in $\mathbb{B P}_{0}$ are perfect, for instance take the Boolean algebra of four elements: $\mathcal{B}_{4} \in \mathbb{B P}_{0}$ but is not perfect. $\mathrm{E}_{3} \times \mathcal{B}_{2}$ (where $\mathrm{E}_{3}$ denotes the MV-algebra of three elements) is a bipartite algebra that is not in $\mathbb{B P}_{0}$. Notice that perfect and bipartite algebras do not have negation fixpoint.

The notions so far presented coincide in the case of IMTL-chains. Indeed we can prove:

Theorem 3.6. Let $\mathcal{A}$ be an IMTL-chain. The following are equivalent:

(1) $\mathcal{A}$ is bipartite.

(2) $\mathcal{A} \in \mathbb{B P}_{0}$

(3) $A=\operatorname{Rad}(\mathcal{A}) \cup \neg \operatorname{Rad}(\mathcal{A})$.

(4) $\operatorname{Rad}(\mathcal{A})=A_{+}$and $\mathcal{A}$ has no fixpoint.

(5) $\mathcal{A}$ is perfect.

(6) $\mathcal{A}$ is isomorphic to the disconnected rotation of a linearly ordered semihoop.

(7) $\mathcal{A}=\left(\neg(\neg x)^{2}\right)^{2} \approx \neg\left(\neg x^{2}\right)^{2}$.

(8) $\mathcal{A} / \operatorname{Rad}(\mathcal{A}) \cong \mathcal{B}_{2}$.

Proof. $(1) \Rightarrow(2),(2) \Rightarrow(3),(3) \Rightarrow(4)$ and $(4) \Rightarrow(5)$ are straightforward.

$[(5) \Rightarrow(6)]$ : If the chain is perfect, then all positive elements have infinite order, hence $A_{+}$is closed under $*$. Therefore, if $\mathcal{B}$ is the prelinear semihoop given by $A_{+}$, then $\mathcal{A} \cong \mathcal{B}^{*}$, since the operation $*$ restricted to $A_{+}$determines the whole operation in $\mathcal{A}$.

$[(6) \Rightarrow(7)]$ : Suppose that there is a totally ordered semihoop $\mathcal{B}$ such that $\mathcal{A} \cong \mathcal{B}^{*}$. An easy computation shows that for every $a \in A_{+}\left(\neg(\neg a)^{2}\right)^{2}=\neg\left(\neg a^{2}\right)^{2}=1$ and for every $a \in A_{-}$ $\left(\neg(\neg a)^{2}\right)^{2}=\neg\left(\neg a^{2}\right)^{2}=0$. 
$[(7) \Rightarrow(8)]$ : Suppose that $\mathcal{A}$ validates the equation. Note that in this case the set of positives is a proper filter. Indeed, if $a \in A_{+}$, then $\neg a \in A_{-}$, so $(\neg a)^{2}=0$. Therefore $\left(\neg(\neg a)^{2}\right)^{2}=1=\neg\left(\neg a^{2}\right)^{2}$ and this implies $a^{2} \in A_{+}$. Now, take $a, b \in A_{+}$such that $a \leq b$. Then $a^{2} \leq a * b$ and $a^{2} \in A_{+}$, so $a * b \in A_{+}$. Thus $A_{+}=\operatorname{Rad}(\mathcal{A})$. Consider the algebra $\mathcal{A} / \operatorname{Rad}(\mathcal{A})$ and take $a \in A$. If a is positive, then $a \rightarrow 1=1 \in \operatorname{Rad}(\mathcal{A})$ and $1 \rightarrow a=$ $a \in \operatorname{Rad}(\mathcal{A})$, so $a / \operatorname{Rad}(\mathcal{A})=1 / \operatorname{Rad}(\mathcal{A})$. If a is negative, then $a \rightarrow 0=\neg a \in \operatorname{Rad}(\mathcal{A})$ and $0 \rightarrow a=1 \in \operatorname{Rad}(\mathcal{A})$, so $a / \operatorname{Rad}(\mathcal{A})=0 / \operatorname{Rad}(\mathcal{A})$. Therefore $\mathcal{A} / \operatorname{Rad}(\mathcal{A}) \cong \mathcal{B}_{2}$.

$[(8) \Rightarrow(1)]$ : Suppose that the quotient by the radical is the two-element Boolean algebra. It will be enough to prove that for every $a \in A(a \in \operatorname{Rad}(\mathcal{A}) \Leftrightarrow \neg a \notin \operatorname{Rad}(\mathcal{A}))$. Indeed: $a$ is in the radical iff $a / \operatorname{Rad}(\mathcal{A})=1 / \operatorname{Rad}(\mathcal{A})$ iff $\neg a / \operatorname{Rad}(\mathcal{A})=0 / \operatorname{Rad}(\mathcal{A})$ iff $\neg a \notin \operatorname{Rad}(\mathcal{A})$.

\subsection{The radical of IMTL-algebras}

In this section we will extend to $\mathbb{I M T L}$ the characterization of the radical given in [15] for MV-algebras.

Lemma 3.7. Let $\mathcal{A}$ be an IMTL-chain. Then:

$$
\operatorname{Rad}(\mathcal{A})=\left\{a \in A: a^{n}>\neg a \quad \forall n \geq 1\right\} .
$$

Proof. If $a \in \operatorname{Rad}(\mathcal{A})$, then for every $n \geq 1, a^{n} \in \operatorname{Rad}(\mathcal{A}) \subseteq A_{+}$. Since $a^{n} \leq a$, we obtain $\neg a \leq \neg a^{n}<a^{n}$. Conversely, take $a \in A$ such that for every $n \geq 1, a^{n}>\neg a$. Then, in particular, for every $n, a^{n} \neq 0$, so the filter generated by $a, \mathbb{F} i(a)$, is proper. Thus, $a \in \mathbb{F} i(a) \subseteq \operatorname{Rad}(\mathcal{A})$, since in chains the filters are totally ordered.

Lemma 3.8. Let $\mathcal{A}$ and $\mathcal{B}$ be IMTL-algebras and $h: \mathcal{A} \rightarrow \mathcal{B}$ a homomorphism.

(a) If $F \subseteq B$ is a filter, then $h^{-1}[F]$ is a filter.

(b) If $F \subseteq B$ is a proper filter, then $h^{-1}[F]$ is a proper filter.

(c) If $F \subseteq B$ is a prime filter, then $h^{-1}[F]$ is a prime filter.

(d) If $F \subseteq B$ is a maximal filter, then $h^{-1}[F]$ is a maximal filter.

(e) If $F \subseteq B$ is a filter, then $h^{-1}[\neg F]=\neg\left(h^{-1}[F]\right)$.

Proof. All the proofs are routine.

Now we can generalize the characterization of the radical to all IMTL-algebras.

Theorem 3.9. Let $\mathcal{A}$ be an IMTL-algebra. Then:

$\operatorname{Rad}(\mathcal{A})=\left\{a \in A: a^{n}>\neg a \quad \forall n \geq 1\right\}$. 
Proof. Suppose first that $a$ belongs to the set on the right side. If there is a maximal filter $M$ such that $a \notin M$, then, by Proposition 2.6 there exists $n \geq 1$ such that $\neg a^{n} \in M$. But, since $a^{n}>\neg a$, we have $\neg a^{n}<a$, so $a \in M$ and this is absurd. To prove the other inclusion, consider a representation of $\mathcal{A}$ as a subdirect product of IMTL-chains, $\varphi: \mathcal{A} \hookrightarrow \Pi_{i \in I} \mathcal{A}_{i}$. For every $i \in I$, let $\varphi_{i}$ be the $i$-th projection of $\varphi$. By the previous lemma, for every $i \in I$, $\varphi_{i}^{-1}\left[\operatorname{Rad}\left(\mathcal{A}_{i}\right)\right]$ is a maximal filter of $\mathcal{A}_{i}$, thus:

$$
\operatorname{Rad}(\mathcal{A}) \subseteq \bigcap_{i \in I} \varphi_{i}^{-1}\left[\operatorname{Rad}\left(\mathcal{A}_{i}\right)\right]
$$

So we only need to prove this inclusion:

$$
\bigcap_{i \in I} \varphi_{i}^{-1}\left[\operatorname{Rad}\left(\mathcal{A}_{i}\right)\right] \subseteq\left\{a \in A: a^{n}>\neg a \quad \forall n \geq 1\right\}
$$

Suppose that for every $i \in I, a \in \varphi_{i}^{-1}\left[\operatorname{Rad}\left(\mathcal{A}_{i}\right)\right]$. Then for every $i \in I, \varphi_{i}(a) \in \operatorname{Rad}\left(\mathcal{A}_{i}\right)$ and, since $\mathcal{A}_{i}$ are chains, for every $n \geq 1,\left(\varphi_{i}(a)\right)^{n}>\neg\left(\varphi_{i}(a)\right)$. Therefore, for every $n \geq 1$, $\varphi(a)^{n}>\neg \varphi(a)$, hence for every $n \geq 1, a^{n} \geq \neg a$. This implies that for every $n \geq 1, a^{n}>\neg a$.

Corollary 3.10. Let $\mathcal{A}$ be an IMTL-chain. Then $\operatorname{Rad}(\mathcal{A}) \subseteq A_{+}$.

Corollary 3.11. Let $\mathcal{A}$ be an IMTL-algebra. Then:

$A_{+}$is a filter if, and only if, $A_{+}=\operatorname{Rad}(\mathcal{A})$.

Proof. Suppose that $A_{+}$is a filter. We have to prove that $A_{+} \subseteq \operatorname{Rad}(\mathcal{A})$. Let $a \in A_{+}$, then for every $n \geq 1 a^{n} \in A_{+}$. We have $a^{n} \leq a$, hence $\neg a \leq \neg a^{n}<a^{n} \leq a$ and this means that $a \in \operatorname{Rad}(\mathcal{A})$.

\subsection{Local IMTL-algebras}

Note that in every IMTL-algebra $\mathcal{A}$, we have: $\operatorname{Rad}(\mathcal{A}) \subseteq\{a \in A: \operatorname{ord}(a)=\infty\}$. We will study now the algebras where this inclusion is an equality, the local IMTL-algebras.

Definition 3.12. An IMTL-algebra $\mathcal{A}$ is local if for every $a \in A, \operatorname{ord}(a)<\infty$ or $\operatorname{ord}(\neg a)<$ $\infty$.

It is clear that chains are local algebras. Also all perfect algebras are local. In fact, we have this characterization:

Proposition 3.13. An IMTL-algebra $\mathcal{A}$ is local if, and only if, it has a unique maximal filter.

Proof. Suppose that $M$ is the unique maximal filter of $\mathcal{A}$. If there is $a \in A$ such that $\operatorname{ord}(a)=\operatorname{ord}(\neg a)=\infty$ then $a, \neg a \in M$ and this is a contradiction since $\mathrm{M}$ is proper. Conversely, suppose that $\mathcal{A}$ is local and let $M$ be a maximal filter. Then it is easy to prove that $M=\{a \in A: \operatorname{ord}(a)=\infty\}$. Clearly $M$ is contained in this set. If $a \notin M$ and $\operatorname{ord}(a)=\infty$, then $\exists n$ such that $\neg a^{n} \in M$, so $\operatorname{ord}\left(\neg a^{n}\right)=\infty$. Hence $\operatorname{ord}\left(a^{n}\right)<\infty$, so $\operatorname{ord}(a)<\infty$, a contradiction. 
Corollary 3.14. Let $\mathcal{A}$ be an IMTL-algebra.

$\mathcal{A}$ is local if, and only if, $\operatorname{Rad}(\mathcal{A})=\{a \in A: \operatorname{ord}(a)=\infty\}$.

There is a special type of filter related to local algebras, namely the primary filters. To define it we need a new operation $\oplus$ in IMTL-algebras, which is the dual operation of $*$ :

Definition 3.15. Let $\mathcal{A}$ be an IMTL-algebra. For every $a, b \in A$ we define $a \oplus b:=\neg(\neg a * \neg b)$, $1 a:=a$ and for every $n>1 n a:=(n-1) a \oplus a$.

Definition 3.16. $P$ is a primary filter of an IMTL-algebra $\mathcal{A}$ if it is a proper filter such that for every $a, b \in A$ such that $a \oplus b \in P$, there is $n \geq 1$ such that na $\in P$ or $n b \in P$.

Theorem 3.17. Let $\mathcal{A}$ be an IMTL-algebra and $P \subseteq A$ a filter. Then:

$\mathcal{A} / P$ is local if, and only if, $P$ is primary.

Proof. Suppose that the quotient is local and take $a \oplus b \in P$ such that for every $n \geq 1$, $n a \notin P$. From one hand, $a / P \oplus b / P=(a \oplus b) / P=1 / P$, hence $\neg(b / P) \leq a / P$. To the other hand, for every $n \geq 1, n(a / P) \neq 1 / P$, so for every $n \geq 1, n \neg(b / P) \neq 1 / P$. It follows that $\operatorname{ord}(b / P)=\infty$, but $\mathcal{A} / P$ is local, so $\operatorname{ord}(\neg(b / P))<\infty$. This implies that there is a $m \geq 1$ such that $m b / P=1 / P$, therefore $m b \in P$. To prove the converse, take an arbitrary $a / P$ and observe that $a \oplus \neg a=1 \in P$. Then, using that $P$ is primary, we have that there is $n \geq 1$ such that $n a \in P$ or $n(\neg a) \in P$. In the first case it is easy to prove that $\operatorname{ord}(\neg a / P)<\infty$, and the second implies $\operatorname{ord}(a / P)<\infty$. Thus, the algebra is local.

Corollary 3.18. Let $\mathcal{A}$ be an IMTL-algebra and let $F \subseteq A$ be a prime filter. Then, $F$ is primary.

Proposition 3.19. Let $F$ be a proper filter of an IMTL-algebra $\mathcal{A}$. Then, $F$ is primary if, and only if, there is a unique maximal filter $M$ such that $F \subseteq M$.

Proof. $F$ is primary iff $\mathcal{A} / F$ is local iff $\mathcal{A} / F$ has a unique maximal filter iff there is a unique maximal filter $M$ such that $F \subseteq M$, since there is an order isomorphism between the filters of $\mathcal{A} / F$ and the filters of $\mathcal{A}$ containing $F$.

Corollary 3.20. An IMTL-algebra is local if, and only if, all its proper filters are primary.

Corollary 3.21. Let $\mathcal{A}$ be a local IMTL-algebra. Then for each filter $F \subseteq A, \mathcal{A} / F$ is local.

Hence, the class of local IMTL-algebras is closed under homomorphic images. It is easy to prove that is also closed under subalgebras. Nevertheless, this class is neither a variety nor a quasivariety since it is not closed under direct products. For instance, the standard nilpotent minimum algebra $[0,1]_{N M}$ is local but the direct product of two copies of it, $\left([0,1]_{N M}\right)^{2}$, is not local. In fact, since all chains are local, the variety generated by the local algebras is the whole IMTL.

In order to state a classification theorem of local algebras, we define two new classes of IMTL-algebras. 
Definition 3.22. An IMTL-algebra $\mathcal{A}$ is locally finite if for every $a \in A \backslash\{1\}$ ord $(a)<\infty$. $\mathcal{A}$ is peculiar if it is local and there are $a, b \in A \backslash\{0,1\}$ such that ord $(a)=\infty$, ord $(b)<\infty$ and $\operatorname{ord}(\neg b)<\infty$.

Locally finite and peculiar algebras can have negation fixpoint; for instance the standard Eukasiewicz algebra on $[0,1]$ is locally finite and $[0,1]_{N M}$ is peculiar, since $\operatorname{ord}\left(\frac{1}{2}\right)=\operatorname{ord}\left(\neg \frac{1}{2}\right)<$ $\infty$.

Theorem 3.23. Let $\mathcal{A}$ be a local IMTL-algebra such that $\mathcal{A} ¥ \mathcal{B}_{2}$. Then $\mathcal{A}$ satisfies one, and only one, of the following:

- $\mathcal{A}$ is perfect.

- $\mathcal{A}$ is locally finite.

- $\mathcal{A}$ is peculiar.

\subsection{Perfect IMTL-algebras and disconnected rotations of prelinear semi- hoops}

For perfect algebras there is also a special type of filters:

Definition 3.24. $P$ is a perfect filter of an IMTL-algebra $\mathcal{A}$ if it is a proper filter such that for every $a \in A$, there exists $n$ such that na $\in P$ if, and only if, for every $m, m(\neg a) \notin P$.

Proposition 3.25. Let $\mathcal{A}$ be an IMTL-algebra and $F \subseteq A$ a proper filter. Then:

$F$ is perfect if, and only if, $\mathcal{A} / F$ is a non-trivial perfect algebra.

Proof. It is straightforward to check.

Corollary 3.26. Every perfect filter is primary.

Proof. By the last proposition and Theorem 3.17.

Theorem 3.27. Let $\mathcal{A}$ be an IMTL-algebra. Then:

$\mathcal{A}$ is perfect if, and only if, every proper filter of $\mathcal{A}$ is perfect.

Proof. If every proper filter is perfect, then $\mathcal{A}$ is perfect since $\mathcal{A} \cong \mathcal{A} /\{1\}$. Now suppose that the algebra is perfect and take an arbitrary proper filter $F \subseteq A$, and $a \in F$. We must prove $(\exists n n a \in F \Leftrightarrow \forall m m(\neg a) \notin F)$. Indeed, $n a \in F \Rightarrow \operatorname{ord}(n a)=\infty \Rightarrow \operatorname{ord}(\neg(n a))<\infty \Rightarrow$ $\operatorname{ord}\left((\neg a)^{n}\right)<\infty \Rightarrow \operatorname{ord}(\neg a)<\infty \Rightarrow \operatorname{ord}(a)=\infty$. If there is $m$ such that $m(\neg a) \in F$, then $\operatorname{ord}(\neg a)=\infty$ and this is absurd.

For the other implication: $\forall m m(\neg a) \notin F \Rightarrow \forall m m(\neg a) \neq 1 \Rightarrow \forall m a^{m} \neq 0 \Rightarrow \operatorname{ord}(a)=$ $\infty \Rightarrow \operatorname{ord}(\neg a)<\infty \Rightarrow \exists n(\neg a)^{n}=0 \Rightarrow \exists n n a=1 \in F$. 
Therefore, we obtain that the class of perfect IMTL-algebras is closed under homomorphic images and obviously is closed under subalgebras. However, considering the subalgebra $\mathcal{A}$ of $[0,1]_{N M}$ generated by $[0,1] \backslash\left\{\frac{1}{2}\right\}$ we obtain that $\mathcal{A}$ is a perfect algebra while $\mathcal{A}^{2}$ is not perfect. So, this class is not closed under direct products.

It is also possible to characterize these algebras in the following way:

Theorem 3.28. Let $\mathcal{A}$ be an IMTL-algebra. Then:

$\mathcal{A}$ is perfect if, and only if, $A=\operatorname{Rad}(\mathcal{A}) \cup \neg \operatorname{Rad}(\mathcal{A})$.

Proof. Suppose that $\mathcal{A}$ is perfect. By Corollary 3.14 we know that $\operatorname{Rad}(\mathcal{A})=\{a \in A$ : $\operatorname{ord}(a)=\infty\}$ and then the result follows immediately. Conversely, if $A=\operatorname{Rad}(\mathcal{A}) \cup \neg \operatorname{Rad}(\mathcal{A})$ then every $a \in \operatorname{Rad}(\mathcal{A})$ has infinite order and every $a \in \neg \operatorname{Rad}(\mathcal{A})$ has finite order, hence the algebra is perfect.

Corollary 3.29. Every perfect algebra is bipartite.

Proof. If the algebra is perfect, then it is local, so the radical is the only maximal filter and the result is obvious.

Another easy consequence is the following proposition about perfect subalgebras:

Corollary 3.30. Given an IMTL-algebra $\mathcal{A}, \operatorname{Rad}(\mathcal{A}) \cup \neg \operatorname{Rad}(\mathcal{A})$ is a perfect subalgebra and contains all perfect subalgebras of $\mathcal{A}$.

Interestingly, the notion of perfect algebra turns out to be the same as the notion of disconnected rotation, as the following theorem proves:

Theorem 3.31. Let $\mathcal{A}$ be an IMTL-algebra. Then the following are equivalent:

(1) $\mathcal{A}$ is perfect.

(2) $\mathcal{A} / \operatorname{Rad}(\mathcal{A}) \cong \mathcal{B}_{2}$.

(3) $\mathcal{A}$ is isomorphic to the disconnected rotation of a prelinear semihoop.

Proof. $[(1) \Rightarrow(2)]$ : If the algebra is perfect, then the radical is perfect and maximal, hence $\mathcal{A} / \operatorname{Rad}(\mathcal{A})$ is simple and perfect, so it must be isomorphic to $\mathcal{B}_{2}$.

$[(2) \Rightarrow(3)]:$ For every $a \in A,(a / \operatorname{Rad}(\mathcal{A})=1 / \operatorname{Rad}(\mathcal{A}) \Rightarrow a \in \operatorname{Rad}(\mathcal{A}))$ and $(a / \operatorname{Rad}(\mathcal{A})=$ $0 / \operatorname{Rad}(\mathcal{A}) \Rightarrow a \in \neg \operatorname{Rad}(\mathcal{A}))$. So $A=\operatorname{Rad}(\mathcal{A}) \cup \neg \operatorname{Rad}(\mathcal{A})$. Then, considering the prelinear semihoop $\mathcal{B}$ given by $\operatorname{Rad}(\mathcal{A})$, we obtain that $\mathcal{A} \cong \mathcal{B}^{*}$.

$[(3) \Rightarrow(1)]$ : If $\mathcal{A} \cong \mathcal{B}^{*}$ for some prelinear semihoop $\mathcal{B}$, then it is obvious that all positive elements have infinite order and all negative elements have finite order.

It is also interesting to particularize this result to MV-algebras: 
Theorem 3.32. Let $\mathcal{A}$ be an IMTL-algebra. The following are equivalent:

(1) $\mathcal{A}$ is a perfect $M V$-algebra.

(2) $\mathcal{A}$ is isomorphic to the disconnected rotation of a cancellative hoop.

Proof. $[(1) \Rightarrow(2)]$ : It is clear that $\operatorname{Rad}(\mathcal{A})$ is a Wajsberg hoop. We only need to show that it has no minimum element and then it will be a cancellative hoop. Suppose that $a$ is the minimum of $\operatorname{Rad}(\mathcal{A})$ and take any $x<a$ different from 0 . Then $a \wedge x=x$, but $a *(a \rightarrow x)=a * \neg(a * \neg x)=\neg(a \rightarrow a * \neg x)=\neg(a \rightarrow a)=0$, a contradiction with $\mathcal{A}$ being a MV-algebra.

$[(2) \Rightarrow(1)]$ : The rotation of a cancellative hoop is always an MV-algebra as it is proved in Lemma 3.13 of [13] and by the last theorem it is perfect.

\subsection{Bipartite IMTL-algebras}

Finally, we will study the class of bipartite algebras. Let $\mathcal{A}$ be a bipartite IMTL-algebra. Recall that these algebras do not have negation fixpoint. It is clear that for every $a \in A$, $\operatorname{ord}(a)=\infty$ or $\operatorname{ord}(\neg a)=\infty$. Take $A_{1}:=\{a \in A: \operatorname{ord}(a)=\infty \Leftrightarrow \operatorname{ord}(\neg a)<\infty\}$ and $A_{2}:=\{a \in A: \operatorname{ord}(a)=\operatorname{ord}(\neg a)=\infty\}$. Then, we have:

- $A=A_{1} \cup A_{2}$.

- $A_{1}=\{0,1\}$ if, and only if, $\mathcal{A} \in \mathbb{B} \mathbb{A}$.

- $A_{2}=\emptyset$ if, and only if, $\mathcal{A}$ is perfect.

Lemma 3.33. Let $\mathcal{A}$ be an IMTL-algebra. We have:

For every $a, b \in A$, if ord $(\neg(a * b))<\infty$, then $\neg a * \neg b=0$.

Proof. It is just an easy computation.

Proposition 3.34. If $A_{1}$ is a subuniverse, then $A_{1}=A_{+} \cup A_{-}$and $A_{+}$is a filter.

Proof. First we prove that for every $a \in A_{1}, \operatorname{ord}(a)=\infty$ implies $a \in A_{+}$. Indeed, if $\operatorname{ord}(a)=$ $\infty$, then $\operatorname{ord}\left(a^{2}\right)=\infty$, so $\operatorname{ord}\left(\neg a^{2}\right)<\infty$ (since $a^{2} \in A_{1}$ ), and thus $\neg a * \neg a=0$ (by the previous lemma), i.e. $a<\neg a$.

Now we check that $A_{1}=A_{+} \cup A_{-}$. Take $a \in A_{1}$. If $\operatorname{ord}(a)=\infty$, then $a \in A_{+}$. If $\operatorname{ord}(a)<\infty$, then $\operatorname{ord}(\neg a)=\infty$, so $\neg a \in A_{+}$and $a \in A_{-}$. Take $a \in A_{+} \cup A_{-}$. If $a \in A_{+}$, then $\operatorname{ord}(\neg a)<\infty$ and $\operatorname{ord}(a)=\infty$, hence $a \in A_{1}$. If $a \in A_{-}$, then $\operatorname{ord}(a)<\infty$ and $\operatorname{ord}(\neg a)=\infty$, hence $a \in A_{1}$.

Finally we prove that $A_{+}$is a filter. It is enough to prove that it is closed under $*$. Take $a \in A_{+}$, then $\operatorname{ord}\left(a^{2}\right)=\infty$, so $a^{2} \in A_{+}$. If $a, b \in A_{+}$, we only need to prove that the order of $a * b$ is infinite. Suppose that it is finite. Then there is $n$ such that $(a * b)^{n}=0$. This implies that there is $m$ such that $(a * b)^{2^{m}}=0$, so $a^{2^{m}} \leq \neg b^{2^{m}}$ but this is a contradiction because $a^{2^{m}}$ and $b^{2^{m}}$ are positive elements. 
Proposition 3.35. Let $\mathcal{A}$ be an IMTL-algebra without negation fixpoint and let $M \subseteq A$ be a prime filter. Then the following are equivalent:

(1) $A_{+} \subseteq M$.

(2) $M$ is maximal and $A=M \cup \neg M$.

(3) $\mathcal{A} / M \cong \mathcal{B}_{2}$.

(4) $M$ is maximal and perfect.

Proof. $[(1) \Rightarrow(2)]$ : If $a \in A$, then by Proposition 2.5, $a \vee \neg a \in A_{+} \subseteq F$, but since $F$ is prime, $a \in F$ or $\neg a \in F$.

$[(2) \Rightarrow(3)]$ : On one hand, $M$ is primary by Corollary 3.18 , so $\mathcal{A} / M$ is local. On the other hand, for every $a \in A, a / M \vee \neg(a / M)=(a \vee \neg a) / M=1 / M$, hence $\mathcal{A} / M$ is Boolean. Therefore, it must be the two-element Boolean algebra.

$[(3) \Rightarrow(4)]:$ If $\mathcal{A} / M \cong \mathcal{B}_{2}$, then $M$ is perfect since $\mathcal{B}_{2}$ is perfect, and $M$ is maximal since $\mathcal{B}_{2}$ is simple.

$[(4) \Rightarrow(1)]$ : Take $a \vee \neg a \in A_{+}$and suppose that is not in $M$. Then there is a $n \geq 1$ such that $\neg(a \vee \neg a)^{n} \in M$, so $n(\neg a) \wedge n a \in M$ in contradiction with $M$ being perfect.

This gives a characterization of bipartite IMTL-algebras:

Theorem 3.36. An IMTL-algebra $\mathcal{A}$ is bipartite if, and only if, the filter generated by $A_{+}$is proper.

Proof. The implication from left to right follows immediately from the last theorem. For the other one, suppose that $\mathbb{F} i\left(A_{+}\right)$is proper. Then it must be contained in some maximal filter $M$, so $A_{+} \subseteq M$ and by the last theorem $A=M \cup \neg M$.

Lemma 3.37. Let $\mathcal{A}$ be an IMTL-algebra without negation fixpoint and let $F \subseteq A$ be a filter. Then: $\mathcal{A} / F \in \mathbb{B} \mathbb{A}$ if, and only if, $A_{+} \subseteq F$.

Proof. Suppose that the quotient is a Boolean algebra and take $a \in A_{+}$. Then $a / F \vee \neg(a / F)=$ $(a \vee \neg a) / F=1 / F$. Thus: $a=a \vee \neg a \in F$. Conversely, it is straightforward to check that $\mathcal{A} / F$ satisfies the law of the excluded middle.

Lemma 3.38. Let $\mathcal{A}$ be a bipartite IMTL-algebra. Then:

$\mathcal{A}$ is bipartite only by $\mathbb{F} i\left(A_{+}\right)$if, and only if, $\mathbb{F} i\left(A_{+}\right)$is a maximal filter.

Proof. Suppose that $\mathbb{F} i\left(A_{+}\right)$is a maximal filter. $A_{+} \subseteq \mathbb{F} i\left(A_{+}\right)$, so $A=\mathbb{F} i\left(A_{+}\right) \cup \neg \mathbb{F} i\left(A_{+}\right)$. If $M$ is a maximal filter and $A=M \cup \neg M$, then $A_{+} \subseteq M$, hence $\mathbb{F} i\left(A_{+}\right)=M$. 
Proposition 3.39. Let $\mathcal{A}$ be a bipartite IMTL-algebra. If $A_{+}$is a filter, then $A_{1}=A_{+} \cup A_{-}$.

Proof. By Lemma 3.37, $\mathcal{A} / A_{+} \in \mathbb{B} \mathbb{A}$. If there exists $a \in A_{1} \backslash\left(A_{+} \cup A_{-}\right)$, then $a / A_{+}$is neither $1 / A_{+}$nor $0 / A_{+}$, so $\operatorname{ord}\left(a / A_{+}\right)=\operatorname{ord}\left(\neg a / A_{+}\right)=\infty$ but this is inconsistent with $a \in A_{1}$ since $\operatorname{ord}(a)<\infty$ or $\operatorname{ord}(\neg a)<\infty$.

Using these results we obtain a good characterization of the algebras in $\mathbb{B P}_{0}$ :

Theorem 3.40. For every IMTL-algebra $\mathcal{A}$ the following are equivalent:

(1) $\mathcal{A} \in \mathbb{B P}_{0}$

(2) $\mathcal{A} / \operatorname{Rad}(\mathcal{A}) \in \mathbb{B} \mathbb{A}$.

(3) $\operatorname{Rad}(\mathcal{A})=A_{+}$and $\mathcal{A}$ has no fixpoint.

Proof. [(1) $\Leftrightarrow(2)]$ : For every maximal filter $M, A=M \cup \neg M$ iff (by Theorem 3.35) $A_{+}$ is contained in every maximal filter iff $A_{+} \subseteq \operatorname{Rad}(\mathcal{A})$. By Lemma 3.37 this is equivalent to $\mathcal{A} / \operatorname{Rad}(\mathcal{A}) \in \mathbb{B} \mathbb{A}$.

$[(2) \Rightarrow(3)]$ : By Lemma 3.37 we obtain $A_{+} \subseteq \operatorname{Rad}(\mathcal{A})$ and the other inclusion is always true.

$[(3) \Rightarrow(2)]$ : Also by Lemma 3.37.

Inside the class of bipartite algebras, this characterization gives:

Proposition 3.41. For every bipartite IMTL-algebra $\mathcal{A}$ the following are equivalent:

(1) $A_{1}$ is a subuniverse.

(2) $A_{+}$is a filter.

(3) $\mathcal{A} \in \mathbb{B P}_{0}$

Proof. $[(1) \Rightarrow(2)]$ : Seen in Proposition 3.34.

$[(2) \Rightarrow(3)]$ : By the last theorem.

$[(3) \Rightarrow(1)]$ : If $\mathcal{A} \in \mathbb{B P}_{0}$, then by the last theorem $A_{+}$is a filter. By Proposition 3.39 we obtain the result.

Corollary 3.42. Every perfect IMTL-algebra is in $\mathbb{B P}_{0}$.

In addition this special class of bipartite algebras is a variety: 
Theorem 3.43. $\mathbb{B P}_{0}$ is a variety. One equational axiomatization of it is obtained by adding the next set of equations to the usual axiomatization for $\mathbb{M M T L}$ :

$$
\left\{(x \wedge \neg x) \rightarrow(x \vee \neg x)^{n} \approx 1: n \geq 1\right\}
$$

Proof. Let $\mathcal{A}$ be an IMTL-algebra. $\mathcal{A} \in \mathbb{B P}_{0}$ iff $\operatorname{Rad}(\mathcal{A})=A_{+}$iff (by Theorem 3.9) for every $a \in A_{+}$and every $n \geq 1 a^{n} \geq \neg a$ iff (by Proposition 2.5) $\mathcal{A}=(x \wedge \neg x) \rightarrow(x \vee \neg x)^{n} \approx 1$ for every $n \geq 1$.

Corollary 3.44. $\mathbb{B P}_{0}$ is the variety generated by all perfect IMTL-algebras, i.e. by all disconnected rotations of prelinear semihoops.

Proof. Let $\mathbb{K}$ be the variety generated by all perfect IMTL-algebras. By Corollary 3.42, $\mathbb{K} \subseteq \mathbb{B P}_{0}$. The other inclusion follows from the subdirect representation theorem and Theorem 3.6 .

There is a simpler axiomatization for $\mathbb{B P}_{0}$ :

Corollary 3.45. $\mathbb{B P}_{0}$ can be axiomatized by adding to the axioms of $\mathbb{I M T L}$ only the following:

$$
\left(\neg(\neg x)^{2}\right)^{2} \approx \neg\left(\neg x^{2}\right)^{2}
$$

Proof. Let $\mathbb{K}$ be the variety of IMTL-algebras satisfying this equation. We will prove $\mathbb{K}=\mathbb{B P}_{0}$. If $\mathcal{A} \in \mathbb{K}$, then by the subdirect representation theorem $\mathcal{A}$ is representable as a subdirect product of chains satisfying the equation. By Theorem 3.6 these chains are in $\mathbb{B P}_{0}$, so $\mathcal{A} \in \mathbb{B P}_{0}$. Conversely, take $\mathcal{A} \in \mathbb{B P}_{0}$. Then $\mathcal{A}$ is isomorphic to a subdirect product of IMTL-chains in $\mathbb{B P}_{0}$, so $\mathcal{A}$ satisfies the equation.

Therefore, we have found a new logic whose algebraic semantics is the variety $\mathbb{B P}_{0}$. We call this logic $B P_{0}$. Obviously, it can be presented in a Hilbert-style calculus by adding the axiom schema $\left(\neg(\neg \varphi)^{2}\right)^{2} \leftrightarrow \neg\left(\neg \varphi^{2}\right)^{2}$ to the calculus given for IMTL. Notice that there are no algebras of $\mathbb{B P}_{0}$ defined over $[0,1]$, since all standard IMTL-algebras have negation fixpoint but perfect algebras do not have it; thus the logic $B P_{0}$ has no standard completeness. Nevertheless, we can prove that this logic has the following Glivenko-style theorem ${ }^{2}$ w.r.t. the classical propositional calculus:

Theorem 3.46. Let $\vdash_{C P C}$ denote the relation of derivability in the classical propositional calculus. Then, for every $\varphi \in F m_{\mathcal{L}}, \vdash_{C P C} \varphi$ if, and only if, $\vdash_{B P_{0}}\left(\neg(\neg \varphi)^{2}\right)^{2}$.

\footnotetext{
${ }^{2}$ For a general study of this type of theorems in the framework of natural expansions of BCK logic see [8].
} 
Proof. Suppose that $\vdash_{C P C} \varphi$. It suffices to prove that for each chain $\mathcal{A} \in \mathbb{B P}_{0}, \mathcal{A} \models$ $\left(\neg(\neg \varphi)^{2}\right)^{2} \approx 1$. Let $\mathcal{A}$ be such a chain and $v: F m_{\mathcal{L}} \rightarrow \mathcal{A}$ an evaluation. We know that $\mathcal{A} / \operatorname{Rad}(\mathcal{A}) \cong \mathcal{B}_{2}$, so $v(\varphi) / \operatorname{Rad}(\mathcal{A})=1 / \operatorname{Rad}(\mathcal{A})$, i.e. $v(\varphi) \in A_{+}$, hence $\left(\neg(\neg v(\varphi))^{2}\right)^{2}=1$. Conversely, if $\vdash_{B P_{0}}\left(\neg(\neg \varphi)^{2}\right)^{2}$, then $\mathcal{B}_{2} \models\left(\neg(\neg \varphi)^{2}\right)^{2} \approx 1$, i.e. $\mathcal{B}_{2} \models \varphi \approx 1$, hence $\vdash_{C P C} \varphi$.

$\mathbb{B P}_{0}$ does not contain all bipartite algebras. So we can ask also for the properties of the class of all bipartite IMTL-algebras.

Proposition 3.47. The class of bipartite IMTL-algebras is closed under subalgebras.

Theorem 3.48. Let $\left\{\mathcal{A}_{i}: i \in I\right\}$ be a set of IMTL-algebras and take their direct product $\mathcal{A}$. If there is $j \in I$ such that $\mathcal{A}_{j}$ is bipartite, then $\mathcal{A}$ is bipartite.

Proof. Using the same reasoning as in Theorem 4.5 of [9].

Corollary 3.49. The class of bipartite IMTL-algebras is closed under direct products.

Corollary 3.50. The variety generated by all bipartite IMTL-algebras is $\mathbb{I M T L}$.

Proof. Let $\mathcal{A}$ be an arbitrary IMTL-algebra. Consider $\mathcal{A} \times \mathcal{B}_{2}$, that is a bipartite IMTLalgebra since $\mathcal{B}_{2}$ is bipartite. Thus, taking the projection over the first component, we obtain $\mathcal{A}$ as a homomorphic image of a bipartite algebra. Therefore, every IMTL-algebra is in the variety generated by all bipartite algebras.

\subsection{Varieties of prelinear semihoops and subvarieties of $\mathbb{B P}_{0}$}

In this section we will prove that the lattice of subvarieties of $\mathbb{B P}_{0}$ is really big and complex. Indeed we show that it is isomorphic to the lattice of subvarieties of prelinear semihoops.

We will need the next notation: given a class of prelinear semihoops $\mathbb{K}$, we define $\mathbb{K}^{*}:=$ $\left\{\mathcal{A}^{*}: \mathcal{A} \in \mathbb{K}\right\}$.

Lemma 3.51. If $\mathbb{K}$ is a class of prelinear semihoops, then $\mathbf{H}\left(\mathbb{K}^{*}\right)=\mathbf{H}(\mathbb{K})^{*}$.

Proof. Take $\mathcal{A} \in \mathbb{K}$ and $\mathcal{B} \in \mathbf{H}(\mathcal{A})$ and consider $\mathcal{B}^{*}$. We know that $\mathcal{B}$ is the image of some homomorphism $h: \mathcal{A} \rightarrow \mathcal{B}$. We must prove that $\mathcal{B}^{*} \in \mathbf{H}\left(\mathcal{A}^{*}\right)$. It suffices to consider the following homomorphism:

$g: \mathcal{A}^{*} \rightarrow \mathcal{B}^{*}$ defined by:

$$
g(a)= \begin{cases}h(a) & \text { if } a \in\left(A^{*}\right)_{+} \\ \neg h(\neg a) & \text { if } a \in\left(A^{*}\right)_{-}\end{cases}
$$

Take now $\mathcal{B} \in \mathbf{H}\left(\mathbb{K}^{*}\right)$, i.e., $\mathcal{B}$ is the image of some homomorphism $h: \mathcal{A}^{*} \rightarrow \mathcal{B}$, where $\mathcal{A} \in \mathbb{K}$. Then $\mathcal{B} \cong h\left[\left(A^{*}\right)_{+}\right]^{*}$, so $\mathcal{B} \in \mathbf{H}(\mathcal{A})^{*} \subseteq \mathbf{H}(\mathbb{K})^{*}$. 
Lemma 3.52. If $\mathbb{K}$ is a class of prelinear semihoops, then $\mathbf{S}\left(\mathbb{K}^{*}\right)=\mathbf{S}(\mathbb{K})^{*}$.

Proof. Take $\mathcal{A} \in \mathbb{K}$ and $\mathcal{B} \in \mathbf{S}\left(\mathcal{A}^{*}\right)$. Since $\mathcal{B}$ is a subalgebra of the disconnected rotation of $\mathcal{A}$, we have that $B_{+} \subseteq\left(A^{*}\right)_{+}=A$ and $B_{-} \subseteq\left(A^{*}\right)_{-}$. Actually, $B_{+}$is the universe of a subalgebra of $\mathcal{A}$ and $\mathcal{B}$ is the disconnected rotation of this subalgebra. Therefore, $\mathcal{A} \in \mathbf{S}(\mathbb{K})^{*}$.

Conversely, if $\mathcal{A} \in \mathbb{K}$ and $\mathcal{B} \in \mathbf{S}(\mathcal{A})^{*}$, then $\mathcal{B}=\mathcal{C}^{*}$ for some $\mathcal{C} \subseteq \mathcal{A}$. It follows that $\mathcal{C}^{*} \subseteq \mathcal{A}^{*}$, so $\mathcal{B} \in \mathbf{S}\left(\mathcal{A}^{*}\right) \subseteq \mathbf{S}\left(\mathbb{K}^{*}\right)$.

Lemma 3.53. If $\mathbb{K}$ is a class of prelinear semihoops, then $\mathbf{P}_{U}(\mathbb{K})^{*} \subseteq \mathbf{I S P}_{U}\left(\mathbb{K}^{*}\right)$.

Proof. Take $\left\{\mathcal{A}_{i}: i \in I\right\} \subseteq \mathbb{K}$ and consider an ultraproduct $\prod_{\mathcal{U}}^{I} \mathcal{A}_{i}$. Consider also the ultraproduct of $\left\{\mathcal{A}_{i}^{*}: i \in I\right\}$ corresponding to the same index set and the same ultrafilter, i.e., $\prod_{\mathcal{U}}^{I} \mathcal{A}_{i}^{*}$. It suffices to take the embedding $\alpha:\left(\prod_{\mathcal{U}}^{I} \mathcal{A}_{i}\right)^{*} \rightarrow \prod_{\mathcal{U}}^{I} \mathcal{A}_{i}^{*}$ defined by:

- If $\bar{a} / \mathcal{U} \in\left(\prod_{\mathcal{U}}^{I} A_{i}\right)_{+}^{*}, \alpha(\bar{a} / \mathcal{U}):=\bar{a} / \mathcal{U}$.

- If $\neg(\bar{a} / \mathcal{U}) \in\left(\prod_{\mathcal{U}}^{I} A_{i}\right)_{-}^{*}, \alpha(\neg(\bar{a} / \mathcal{U})):=\neg \bar{a} / \mathcal{U}$, where for every $i \in I(\neg \bar{a})(i)=\neg(\bar{a}(i))$.

We obtain $\left(\prod_{\mathcal{U}}^{I} \mathcal{A}_{i}\right)^{*} \in \mathbf{I S}\left(\prod_{\mathcal{U}}^{I} \mathcal{A}_{i}^{*}\right) \subseteq \mathbf{I S P}_{U}\left(\mathbb{K}^{*}\right)$

Lemma 3.54. If $\mathbb{K}$ is a class of prelinear semihoops, then $\mathbf{P}_{U}\left(\mathbb{K}^{*}\right) \subseteq \mathbf{I S}\left(\mathbf{P}_{U}(\mathbb{K})^{*}\right)$.

Proof. Take $\left\{\mathcal{A}_{i}: i \in I\right\} \subseteq \mathbb{K}$ and an ultraproduct $\prod_{\mathcal{U}}^{I} \mathcal{A}_{i}^{*}$. Given $\bar{a} \in \prod^{I} A_{i}^{*}$, we define $j(\bar{a}) \in \prod^{I} A_{i}$ as:

$$
j(\bar{a})(i):= \begin{cases}\bar{a}(i) & \text { if } \bar{a}(i)>\neg \bar{a}(i), \\ \neg \bar{a}(i) & \text { otherwise. }\end{cases}
$$

In order to show that $\prod_{\mathcal{U}}^{I} \mathcal{A}_{i}^{*} \in \mathbf{I S}\left(\left(\prod_{\mathcal{U}}^{I} \mathcal{A}_{i}\right)^{*}\right) \subseteq \mathbf{I S}\left(\mathbf{P}_{U}(\mathbb{K})^{*}\right)$ it is enough to consider the embedding $\alpha: \prod_{\mathcal{U}}^{I} \mathcal{A}_{i}^{*} \rightarrow\left(\prod_{\mathcal{U}}^{I} \mathcal{A}_{i}\right)^{*}$ defined by:

- If $\bar{a} / \mathcal{U} \in \prod_{\mathcal{U}}^{I} A_{i}^{*}$ is such that $\{i \in I: \bar{a}(i)>\neg \bar{a}(i)\} \in \mathcal{U}$, then $\alpha(\bar{a} / \mathcal{U}):=j(\bar{a}) / \mathcal{U}$.

- If $\bar{a} / \mathcal{U} \in \prod_{\mathcal{U}}^{I} A_{i}^{*}$ is such that $\{i \in I: \bar{a}(i)<\neg \bar{a}(i)\} \in \mathcal{U}$, then $\alpha(\bar{a} / \mathcal{U}):=\neg \alpha(\neg \bar{a} / \mathcal{U})$.

Theorem 3.55. Let $\mathbb{K}$ and $\mathbb{L}$ be classes of totally ordered prelinear semihoops. Then, $\mathbf{V}(\mathbb{K})=$ $\mathbf{V}(\mathbb{L})$ if, and only if, $\mathbf{V}\left(\mathbb{K}^{*}\right)=\mathbf{V}\left(\mathbb{L}^{*}\right)$.

Proof. From Jónsson's Lemma (see [4]) we deduce that given a class $\mathbb{M}$ of prelinear semihoops or IMTL-algebras, $\mathbf{H S P}_{U}(\mathbb{M})$ coincides with the class of the chains in $\mathbf{V}(\mathbb{M})$. Therefore, due to the representation in subdirect products of chains, we only need to prove: $\mathbf{H S P}_{U}(\mathbb{K})=$ $\mathbf{H S P}_{U}(\mathbb{L})$ iff $\mathbf{H S P}_{U}\left(\mathbb{K}^{*}\right)=\mathbf{H S P}_{U}\left(\mathbb{L}^{*}\right)$. 
Suppose first that $\mathbf{H S P}_{U}(\mathbb{K})=\mathbf{H S P}_{U}(\mathbb{L})$. Therefore, $\mathbb{K} \subseteq \mathbf{H S P}_{U}(\mathbb{L})$, so $\mathbb{K}^{*} \subseteq\left(\mathbf{H S P}_{U}(\mathbb{L})\right)^{*}=$ $\mathbf{H S}\left(\mathbf{P}_{U}(\mathbb{L})\right)^{*} \subseteq \mathbf{H S I S P}_{U}\left(\mathbb{L}^{*}\right) \subseteq \mathbf{H S P}_{U}\left(\mathbb{L}^{*}\right)$. Thus, $\mathbf{H S P}_{U}\left(\mathbb{K}^{*}\right) \subseteq \mathbf{H S P}_{U}\left(\mathbb{L}^{*}\right)$, and the other is inclusion is provable in the same way.

Suppose now that $\mathbf{H S P}_{U}\left(\mathbb{K}^{*}\right)=\mathbf{H S P}_{U}\left(\mathbb{L}^{*}\right)$. Then, $\mathbb{K}^{*} \subseteq \mathbf{H S P}_{U}\left(\mathbb{L}^{*}\right) \subseteq \mathbf{H S I S}\left(\mathbf{P}_{U}(\mathbb{L})\right)^{*}=$ $\left(\mathbf{H S I S P}_{U}(\mathbb{L})\right)^{*} \subseteq\left(\mathbf{H S P}_{U}(\mathbb{L})\right)^{*}$. Therefore, $\mathbb{K} \subseteq \mathbf{H S P}_{U}(\mathbb{L})$, and the other inclusion is provable in the same way.

\section{Concluding remarks}

We have done some first steps in the general study of the variety $\mathbb{I M T L}$, the algebraic counterpart of involutive fuzzy logics. ${ }^{3}$ By generalizing the concepts of perfect, local and bipartite algebra defined formerly for MV-algebras we have characterized several classes of IMTL-algebras. In particular, we have given a new insight to Jenei's disconnected rotation presented in [20] showing that the algebras obtained by this method are exactly the class of perfect IMTL-algebras. Futhermore, we have studied the variety generated by those algebras and we have proved that it is $\mathbb{B P}_{0}$. This gives, of course, a new fuzzy logic, namely the extension of IMTL obtained by adding the axiom $\left(\neg(\neg \varphi)^{2}\right)^{2} \leftrightarrow \neg\left(\neg \varphi^{2}\right)^{2}$. However, this logic has no standard completeness, i. e. is not complete w.r.t. the semantics given by the algebras over $[0,1]$ because there are no IMTL-algebras over $[0,1]$ without negation fixpoint.

Notice that $\mathbb{B P}_{0}$ is much bigger than the variety obtained in the case of MV-algebras. Indeed, the variety generated by perfect MV-algebras has only one proper non-trivial subvariety, the class of Boolean algebras, while our $\mathbb{B P}_{0}$ in $\mathbb{M M T L}$ has infinitely many (for instance, all the varieties of NM-algebras without fixpoint classified in [16]) and, moreover, this lattice of subvarieties is isomorphic to the lattice of varieties of prelinear semihoops. This strong difference suggests that the study of the structure of subvarieties of $\mathbb{B P}_{0}$, and in general of $\mathbb{I M T L}$, will be a deep and challenging task.

Acknowledgements: The authors acknowledge partial support of the Spanish projects TIN2004-07933-C03-01, TIN2004-07933-C03-02 and MTM 2004-03102 and the Catalan project 2001SGR-0017 of DGR. We are also indebted with professors Antonio Di Nola and Brunella Gerla from the University of Salerno for their useful comments and for providing excellent research conditions during the visit of the first author in their university on the winter of 2004.

\section{References}

[1] R. Ambrosio and A. Lettieri. A classification of bipartite MV-algebras. Math. Japonica 38, No. 1 (1993) 111-117.

[2] L.P. Belluce, A. Di Nola, A. Lettieri. Local MV-algebras. Rend. Circ. Mat. Palermo (2) 42 (1993) 347-361.

\footnotetext{
${ }^{3}$ Some varieties of IMTL-algebras had been already studied in [7], [16] and [17].
} 
[3] W. J. Blok and D. Pigozzi. Algebraizable logics. Mem. Amer. Math. Soc. 396, vol $77,1989$.

[4] S. Burris and H. P. Sankappanavar. A course in Universal Algebra. Springer Verlag, New York, 1981.

[5] C.C. Chang. Algebraic analysis of many valued logics, Trans. Amer. Math. Soc. 88 (1958) 456-490.

[6] C.C. Chang. A new proof of the completeness of the Lukasiewicz axioms, Trans. Amer. Math. Soc. 93 (1959) 74-80.

[7] A. Ciabattoni, F. Esteva and L. Godo. T-norm based logics with $n$-contraction. Neural Network World 5 (2002) 441-452.

[8] R. Cignoli and A. Torrens. Glivenko like theorems in natural expansions of BCKlogic. Mathematical Logic Quarterly 50(2) (2004) 111-125.

[9] A. Di Nola, F. Liguori and S. Sessa. Using maximal ideals in the classification of MV-algebras. Portugaliae Mathematica 50 (1993) 87-102.

[10] A. Di Nola, S. Sessa, F. Esteva, L. Godo and P. Garcia. The variety generated by perfect BL-algebras: an algebraic approach in a fuzzy logic setting, Annals of Mathematics and Artificial Intelligence 35 (2002) 197-214.

[11] F. Esteva, J. Gispert, L. Godo and F. Montagna. On the standard and Rational Completeness of some Axiomatic extensions of Monoidal t-norm Based Logic. Studia Logica 71 (2002) 199-226.

[12] F. Esteva and L. Godo. Monoidal t-norm based Logic: Towards a logic for leftcontinuous t-norms. Fuzzy Sets and Systems 124 (2001) 271-288.

[13] F. Esteva, L. Godo, P. Hájek, F. Montagna. Hoops and Fuzzy Logic. J. Logic Computat. Vol. 13 No. 4 (2003) 531-555.

[14] J. Fodor. Nilpotent minimum and related connectives for fuzzy logic, Proc. FUZZIEEE'95, 1995, pp. 2077-2082.

[15] J. M. Font, A. J. Rodríguez, A. Torrens. Wajsberg Algebras. Stochastica Vol. VIII 1 (1984) $5-31$.

[16] J. Gispert. Axiomatic extensions of the nilpotent minimum logic. Reports on Mathematical Logic 37 (2003) 113-123.

[17] J. Gispert, A. Torrens. An approach to the variety IMTL(3). Mathematics preprint series, Universitat de Barcelona 351 (2004).

[18] P. HÁJEK. Metamathematics of Fuzzy Logic, Trends in Logic, vol. 4 Kluwer, 1998.

[19] U. HöHLE. Commutative, residuated l-monoids. In: U. Höhle, P. Klement eds., NonClassical Logics and their Applications to Fuzzy Subsets, Kluwer Acad. Publ., Dordrecht 1995, 53-106. 
[20] S. JENEI. On the structure of rotation-invariant semigroups. Archive for Mathematical Logic 42 (2003) 489-514.

[21] S. JenEI. How to construct left-continuous triangular norms - state of the art. Fuzzy Sets and Systems 143 (2004) 27-45.

[22] Y. Komori. Super-Lukasiewicz Propositional Logics. Nagoya Math. J. Vol 83 (1981) 119-133. 